Peter Havaj*

\title{
THE QUALITY AND THE COMPLETE EVIDENCE SECURING DURING THE TRAFFIC CRIME SCENE INVESTIGATION AND ITS RELEVANCE FOR EVIDENCE COMPLETION DURING THE TRAFFIC ACCIDENTS
}

Main purpose of this paper is to point out the problems considering the modern scientific usage of methods, ways and approaches, including the crime investigation of traffic accidents-collisions. We want to show the basic need of experienced traffic crime detective, his/her deep knowledge of the whole issue - the process of the traffic accident perpetrating as a complex process with the direct impact on the traffic crime detective work, which could be used in the process of the clearance the case, the video-record output created by program PC CRASH as the virtual element of legal evidence, enabling deeper knowledge of the whole process.

Keyword: traffic accident-collision, crime scene investigation, error elimination, process of gaining the legal evidence

\section{Introduction}

Traffic accident is an event in the traffic and it represents special, complex and undivided issue from the technical point of view and the next legal considerations. The results are derived directly from the quality and complete evidence securing, which represents crime scene investigation - as the specific method of criminal clearance, based on direct observation, exploring and evaluation of the material environment, its separate objects related to the criminal case with the main objective to clear the case, to seek and to secure the criminal evidence meant to serve as the prodigy to objective truth and to its perpetrator, as the basic need for detective's next approach [1].

Basic tactical principles of the crime scene investigation (CSI):

- crime scene investigation is managed by one leader - CSI is a team work, including the work of police officers, detectives, tech-lab unit, specialists and other partake persons. This group has to set certain objectives and must take the professional responsibility for the whole process and its results.

- CSI urgency - it is derived from the time change of the specific criminological trails due to natural aging process and also due to external influence of the environment. The CSI urgently eliminates these changes or contamination of these criminal evidence.

- CSI non-repeating - the inadmissibility of the CSI recurrence of result from the fact that the probative crime evidence value could be easily objected in the court. The CSI repeating shows negligence and irresponsible approach. Value of the gained evidence during the repeated CSI is generally lower.

- CSI cannot be substituted - it is derived from the significant principle that emerges from the fact that the CSI is separate criminalist methods. Its results cannot be substituted by other means of observation and evaluation.
Traffic criminal scene investigation (TCSI) - meaning, methods, perspective research and error elimination

The TCSI is the first step to achieve adequate investigation efficiency as the final relevant and accurate legal consideration.

The basic principle of the TCSI is to collect facts, which have tactical and legal meaning. The TCSI includes methods and drills as the measuring, which provide the main conclusions.

Meaning of the TCSI is based on seeking, finding, identifying, securing of the criminal evidence or other trails, which have special meaning for identifying the traffic accident reasons.

The results of the TSCI are documented by:

Photo-document collection or video record

TSCI statement

- Sketch (graphical description) of the traffic accident site, which serves to prepare a final plan of the traffic accident site.

Within the theory of the forensic tactics, the TSCI uses basis methods of measuring and tracking the position on the crime scene. They can be divided into:

a. Direct methods, which are touchable and untouchable, touchable mean direct measuring by measuring tools and untouchable mean laser scanning, photogrametry etc.

b. Indirect methods (for example digitalization).

Important elements in space characteristic are: section shape of the accident road section and terrain lengthwise profile of the accident road section and terrain cross profile of the accident road section and terrain

\section{Other elements that influence the quality of the TCSI result}

Besides the equipment, the main predispositions of the high level quality of the TCSI are work experiences, knowledges and responsiblity of investigators. They have to recognize the crime

\footnotetext{
* Peter Havaj

University of Security Management in Kosice, Slovakia

E-mail: peter.havaj@vsbm.sk
} 
evidence, they have to know where it can be found and how to secure and document it. It is necessary to understand, that the TSCI is not a simple act. The TSCI is carried out in different weather and environment conditions, the factor of time is also important. The quick and wise decisions during the TSCI is very important for next steps, on the other hand, an incorrect decision can significantly complicate further investigations. Inexperience and little knowledge of the detectives are the main reasons of spoiling or rather complete destruction of the evidence provided by the TCSI for the following legal process.

The TSCI often follows after the medical emergency or firefighter action. It contains rescuing persons from the damaged vehicle, emergency reviving of the victims, preventing of fire situation, gas loosing, contamination. These actions deteriorate the status of the next TSCI, for example victims are not in their original places, so the detecting of original location has to be done later.

Cleaning the road from the debris, cutting or moving cars could also change the traffic accident site.

Position changes, degradation of crime evidence and other clues, after the medical emergency or firefighter action, change the original status of the TCSI. It can complicate not only the TSCI but later analysis, as well. Minimizing the degradation process due to medical emergency or firefighter action can be realized by preliminary criminal photo collection carried out by rescue services.

\section{Error elimination during archiving TCSI}

The point of measuring (POM) is chosen by subjective stance of the detective and sometimes it is not specifically precise (beginning of the road curve). In such a case a possibility for great difference in the POM description could be created. Occasionally, the selected POM cannot be found in time, since the object was damaged or removed (e.g. a traffic sign). Problem of "close POM" forces detective to recognize the POM before and after the measuring, which can lead to wrong interpretations. The distant POM can be also a problem (several hundred meters), which decreases the precision of the later measuring. It is helpful to create auxiliary point of measuring (APOM), which is precisely localized with respect to the POM and other measurements can be drafted from this point. The localisation of the whole TCSI site should include the GPS, especially for accidents in the forest or field. Irreplaceable error eliminator is high level crime photo collection, which enables later additional photogrammetric identification of the evidence, that was previously neglected on site. The crime photo collection must be presented for the later process in electronic form with a HD, because the printed form cannot show the high level zoom and details.

The crime photo collection in the highlight quality (resolution $640 \mathrm{x} 480$ ) is not appropriate for photogrammetric examination. The crime photo-documentation must, in particular, show vehicle damage indetail and traces on them and other objects and enable to create the entire image of the accident site, individual shots have to follow. The TCSI also includes the cargo weight, pedestrian, bicycle and motorcycle rider weight and height. Those information should be a part of the medical report of the injured persons, as well as the strict localisation of the injuries (for example broken pedestrian leg focused from heel represents decisive point of the accident process). The TCSI also includes detection of fastening belts and airbags status as well as the status of the vehicle interior (seat deformation, broken and deformed parts of the interior).

Those facts are important for the next clearance, as well as the vehicle technical parameters. Special issue of the TCSI are traffic accidents during the bad weather and poor visibility (fog, snowing, heavy rain etc.), where localisation must be connected with the observatory description, because it is very important to distinguish for example unilluminated person or vehicle lights.

Public lights and their location, intensity and functionality in specific time are very important information for the whole legal process and its results. Error elimination also includes the position of possible unknown vehicle, which obstructed the view of the driver, but which already has left the TCSI. In that case, it is important to localise such a vehicle by statement of each witness. During the collisions of moving and standing vehicles is necessary to localise original position of the stationary vehicle before collision. It is important during the chain crashes.

The next removable imprecision is determination of lights condition at the time of traffic accidents, which can be determined at the time of detectives' arrival. It is obvious that light conditions during a certain day period can be changed within minutes (e.g. sunset) and this fact has to be also taken into account. In addition, the impact of lights of opposing vehicles has to be realistically evaluated, for example determination of the pedestrian, who was illuminated outside the range of the car lights. The crime photo collection of the car lights range and visibility is realized during the night time, without using photo flash or other source of light. It is necessary to point out the difficulty and complexity of the TCSI, which includes mistakes, errors and also necessity to eliminate them in all available ways, where for example could be included the detective's experiences, technical inventory and cooperation with emergency and firefighter units. The training of the traffic detectives should also include audit of quality of special tasks and activities during the TCSI.

Main tool that influences the effectiveness of the traffic accident investigation, is interrogation of participants and witnesses of the traffic accident. Its objectives are:

- clarification of the traffic accident, which was subjectively perceived and observed by the interrogating person.

collecting other information that have significant influence on the traffic accident (cargo weight, personal motoric skills, height and injuries) from the witness' point of view

- activities of a witness during the crash accident (reaction, reaction after the crash - breaking, turning the wheel)

finding the specific visual and acoustic perception of the witness ("I've seen")

seeking the circumstances that might have influence on a traffic accident (illumination, covered driver's view, special objects)

securing such subjective information from the interviewers, which can be confirmed by subsequent evidence or can be excluded

- finding everything 
- securing special subjective information from the witness which can exclude intentional changes in later testimony in front of the court

For securing the quality and complete interview, an analysis of the mistakes in this process was created and, therefore, the PC program "Interrogation" was created, which provides that:

- asked questions will be according to the roster of the recommended questions

- $\quad$ it is possible to add questions into the roster according to the specific conditions of the road accident section and other local specifics

- $\quad$ it is possible to add questions based on detective's experience. Injuries and death of persons, as a result of the traffic accident, belong to the group of injuries characterized by specific signs and these signs are reflected in the process of documentation, evidence and legal considerations.

One of the signs is that the occurrence of injuries to road accident participants is usually directly related to the movement of vehicles that are complex technical equipment moving on the road (usually the road) and whose movement is fully subject to physical law. Vehicles during the collision leaves traces, they become deformed, they stop in final position, which is objectively documented, measured and detectable. If a vehicle crash does not involve injury and death, it can be considered as a technical problem [2].

Situation is different when the traffic accident involves human or pedestrians. Human has different parameters of movement from the technical point of view, he/she has different body properties, capability of movement, capability of speed change and geometrical shape (fall, inclination etc.). In that case, it is necessary to add the coroner - forensic surgeon considerations to technical considerations. Human body is always severely damaged, which reflects the collision and possible tracks. Forensic exploration then begins at the stage of measuring and documenting of the traces and then proceeds to assess the cause and the way they are generated [2].

The forensic surgeon examination is considered as a special action, which demands special preparation and competence. It is known that most of the MD's are capable, but less capable in this concrete task. The problem is caused by the absence of technical knowledge resulting to the non complex evaluation or misinformation about the results of technical evidence.

This scientific paper brings a selected range of issues to expand the knowledge and aims which contribute to:

- clarify the possibilities for interdisciplinary procedures, both by technical analysts and by forensic practitioners

- to show value of the physical evidence found on the human body and its meaning during investigation from the specialist point of view and also from the detectives point of view, who coordinate the whole process

- to clarify the value of evidence found on the body and their counterfeit value by the courts for the purposes of legal assessment of the course of a traffic accident.

It is obvious that special forensic procedures and knowledge should be defined according to science discipline - biochemistry, which includes the main procedures.

\section{Pedestrian injuries in traffic accident - criminal tracks and evidence}

Pedestrian injuries caused by traffic accident can be evaluated by:

I. Legal examination

It is derived from the source of injury and final effect of definition needed for the TCSI, proving the guilt and measure of the caused damage for financial retribution of the affected persons.

\section{Medical evaluation}

It is derived from the possibility of diagnostics and the following healing process of injuries and from the principle of reducing the health consequences, as well as quantifying the extent of injury for the purpose of compensating the injured person.

III. Forensic surgeon examination

a. Approaches the assessment of injuries for subsequent legal proceedings in the context of an investigation of the facts

b. Evaluates the injuries for interdisciplinary evidence, measures the violence and its impact on the body of the injured, as well as the detailed localization of the places where the violence was inflicted on the body (system FORTIS).

Forensic surgeon examination can be divided into: assessing of injuries to persons who have not survived the traffic accident

- $\quad$ assessing of injuries to persons who have survived the traffic accident.

Observation of the injured body (OIB) can be considered as the part of the TSCI not realized on the place of traffic accident. It can be considered as later observation of the injured body (LOIB).

Output of the LOIB is crime photo collection, description and medical documentation.

The traffic accident is a unique action according to physics, unrepeatable system of phenomena, influenced by many factors, absolutely individual for each traffic accident. The best way to get clearance of the TSCI is usage of all available knowledge and relevant actions. Said simple, more information - better results [3].

Traces of victims and injured persons are integral part of an accident and are the main sources of information about its course - especially in the collision phase, and has to be a part of the accident analysis as well as expert evidence. Evaluated criminal traces by forensic surgeons are objectified, localised and parameterized for the traffic specialist. In some cases analysts send the information to forensic surgeons for assessment the circumstances that caused the injuries, [4].

Pedestrian injury can be considered as criminal evidence for the TSCI. For this purpose, the system FORTIS is created, which allows the forensic surgeon assessment to be used for the needs of the accident analysis as an equivalent to the other traces on the vehicle, the roadway or other objects. The evaluation of the FORTIS injury then represents de facto the forensic footprint sophisticated judiciary assessment (see the following definition of a trace from the point of view of general criminal activity). Using 
the appropriate methods allows to include injuries occurring during traffic accident between the criminal evidence.

Criminal evidence is every change in material space of criminal relevant action, which can be related by causality, time or place and it can be findable and usable in the process of clearance.

Necessary condition - creation of the criminal evidence in causality with the criminal deed

Need of findability, seekability, securability and usability is derived from the practical need of change as how can criminal evidence be found, kept, distinguished.

Meaning and value of criminal evidence - the criminal trace is useful only in the case that it can be decoded and used. This can be done when one is possible to find, secure and examine the traces by technical means and method.

Each CSI evidence has its value [5]:

- The criminological-tactical value of criminal footprints is particularly applicable in assessing the way in which criminalistic relevant events are committed. Tactical value of the crime evidence is applied in considerations of the criminal ways of objects related to the TSCI. Information value of the crime evidence enables considering the circumstances of the origin, course and extinction of the relevant criminal events, causes and conditions that enabled it.

- Technical value of the crime evidence is related to the possibility of its finding, capturing and later examination

- Legal value of the crime evidence means that legally obtained crime evidence has a special place in the legal process and may have the value of proof in criminal proceedings under some conditions.

Evaluation of the person's injuries includes methods of forensic surgeon examination, classification and use for evidence.

Currently, one can divide classification, identification, as well as considerations of injuries as crime evidence, from the forensic point of view, into four basic evolution levels [6], [7]:

\section{The 1st level}

Typical examination exercised by descriptive way with the usage of minimal, almost none exact methods, based mostly on the specialist "experiences". The possibility of misconduct by forensic practitioners is mainly related to their technical ignorance of the actual course of the accident and the possibility of proving beyond "convincing" is none and relies primarily on the confidence in the knowledge of the forensic practitioner and the correctness of his judgment.

\section{The 2nd level}

Comparison connected with the simple modeling of the damaged body movement, or general consultation with tech specialist without closer explaining of the individual aspects of created injuries. The possibility of erroneous conclusions is partially eliminated, but produced evidence is based mostly on the expert's "experience and belief" and relies on the confidence in the knowledge of the forensic practitioner and on the correctness of his judicial assessment.

\section{The 3rd level}

Comparison connected to cooperation with the technical specialist in such a manner, that visually compares the process of the damaged body movement calculated in a simulation program (video calculation of collision).

Possibility of the forensic surgeon wrong results are mostly eliminated, but the crime evidence is still bound to the experience of the specialist, as well as the way of his calculations of the collision, where the exact knowledge about injuries is not used and the way of its creation.

\section{The 4th level}

A detailed description of the identified injuries and their localization by using documented traces (measurements, photodocumentation) and defining the findings in a standardized form applicable to a technical expert (e.g. the FORTIS system) as a description of the traces, which has to be taken into account in the calculation of collision and to document their compliance with the judgment finding followed by consultations of the forensic practitioner and technical expert and evaluation of the results of the video presented and review of the contact parameters. The possibility of false conclusions is virtually eliminated and this procedure leads to the fact that the obtained evidence has all the features of accuracy, completeness and controllability in relation to all found traces.

Crime evidence of the 4th level was repeatedly accepted from courts of justice in criminal and civil cases.

\section{Forensic surgeon system for parameterization and localisation of injuries by FORTIS and its possibility for crime evidence usage}

Nowadays, the forensic surgeon analysis for traffic accident injuries does not always follow a standard line, due to the absence of certain procedures for standardization of injuries.

The classic way of evaluation the injuries is a scale of Abbreviated Injury Scale/Injury Severity Score (hereinafter AIS/ ISS) derived from medical considerations [8].

Other systems are GSI (Gadd System Index), HIC (Head Injury Consideration), 3MS, TTI (Chest Injury), VC (Soft Tissue dDamage), EIC (Broader Injuries). These parameterization systems do not provide sufficient information for the crime evidence standard, because they are derived from the effect of the injury, not the cause. Forensic surgeons recognize cause of the violence and divide it into low, medium, and high category (for example low speed of the vehicle) without closer specifications. It is also important to consider the fact that not all the damage to health of a pedestrian is a direct consequence of the degree of violence on his body during the collision and then the question arises if the death has been caused by the direct impact of the collision with the vehicle [4].

Mentioned deficiencies of existing status are solved by a modified system FORTIS (Forensic Traumatology Injury Scale), which enables a more comprehensive assessment of the severity in Fortis points (FP) injury and also distinguishes the causes of health damage in the following categories:

BI - basic injuries (direct impact of violence)

Co1 - direct post-traumatic complications (e.g. traumatic shock, hemorrhagic shock, cardiac tamponade, hemothorax, pneumothorax) 


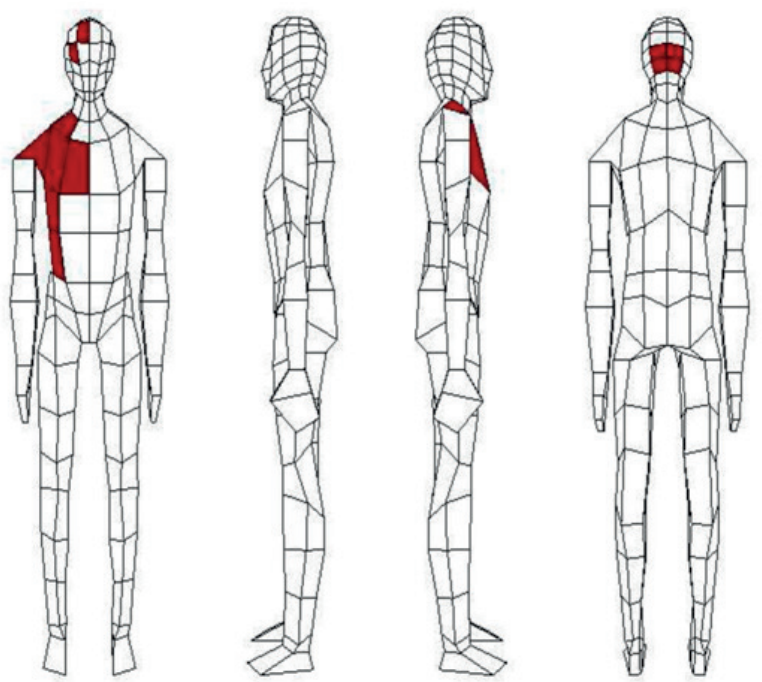

Figure 1 Visualization of the FORTIS output for injury classification after a real traffic accident according to description X [11]

Co2 - associated complications (e.g. inflammatory changes, edema of non-traumatic etiology, thrombosis, conditions after surgery, etc.)

Methodology of parameter standardisation of the pedestrian injuries in traffic accidents (quantification of injuries) requires application of the self-modified system FORTIS, which enables (with high level input parameters) to calculate the severity of the underlying health damage, direct post-traumatic complications and associated complications, including more painful treatment and poor quality health care, and in the case of fatal injuries determines the health damage that is the direct cause of death [9].

Mentioned classification of the health damages can be considered significant in legal assessment.

According to the documentation and the TCSI it is ideal to use PC FORTIS, which was designed for easier drills. Except for patient data (age, weight, clothes, diagnosis description), it enables direct localisation the causes of the injuries by saved data.

\subsection{PC Fortis in action}

$\mathrm{X}$ - description of person's injuries [10]:

- bruises and bloody skin existing on the head, chest and abdomen

- damaged abdomen (mentioned in medical documentation)

serial bone fractures of the 3rd to 8th right ribs

- closed bone fracture of clavicula with removed part of the bone at the right

damaged right lung,

- damaged brain core in the middle brain area (according to MRI)

- diffusional anoxal damage of the brain (according to MRI)
Table 1 Visualisation of FORTIS@ - score FORTIS in BF output of every part of the injured body according to medical examination [11]

\begin{tabular}{ccccc}
\hline & total & ZPZ & Ko1 & Ko2 \\
Hull & 14.2 & 14.2 & 0 & 0 \\
\hline Pelvic bone & 0 & 0 & 0 & 0 \\
Right thigh & 0 & 0 & 0 & 0 \\
Right calf & 0 & 0 & 0 & 0 \\
Right foot & 0 & 0 & 0 & 0 \\
Left thigh & 0 & 0 & 0 & 0 \\
Left calf & 0 & 0 & 0 & 0 \\
Left foot & 0 & 0 & 0 & 0 \\
Left upper arm & 0 & 0 & 0 & 0 \\
Left forearm & 0 & 0 & 0 & 0 \\
Right upper arm & 1 & 1 & 0 & 0 \\
Right forearm & 0 & 0 & 0 & 0 \\
Neck & 0 & 0 & 0 & 0 \\
Head & 7.6 & 7.6 & 0 & 0 \\
Left knee & 0 & 0 & 0 & 0 \\
Right knee & 0 & 0 & 0 & 0 \\
\hline FORTIS total ZPZ & 22.8 & & & \\
FORTIS total Ko1 & 0 & & &
\end{tabular}

\subsection{Output of the FORTIS}

Output of FORTIS is localisation of every injury (see description $\mathrm{X}$ ), final point value, point value according to cause and creation of the injury and its location on the human body (Figure 1).

Detailed description score according to the FORTIS is compatible to the system PC CRASH, which calculates the physical parameters of the contacts and enable to distinguish the violence during the collision, which has affected individual parts of it, respectively recognizing the consequences of this violence on the pedestrian, which uses the physical parameters [9].

System Fortis represents practically usable tool for replacing medical terminology for the needs of experts and traffic accident analysts, with a description of the injuries expressed in a joint statement by their parameterization with sufficient counter value [8].

Program PC FORTIS can be considered as a guide line for specialist and detective practitioners. System FORTIS in parameterization of injuries enables the direct visualization of the injury display, as a significant information for the road traffic expert, and can be considered as an integral part of the solution of pedestrian injury parameterization. System also allows not only to visualize the location of the injuries but to create an individual characteristics of injury, as well (the ratio of violence acting on individual parts of the body according to its distribution according to the computational model in the PC Crash simulation program used for the accident analysis), for specific collisions and conditions, including o.i. the individual characteristics of the pedestrian's body, its position, movement, type of vehicle, its speed, dynamics at the moment of collision, etc. [9]. 
Detective must apply coordination of interdisciplinary evidence. Detective has clearly to understand the method of evidence, the value of their outcomes and the overall complexity and usability for the needs of the legal assessment.

The use of the FORTIS system for the needs of an accident analysis provide a procedure for the analysis of a forensic practitioner and a judicial expert, which aims to:

- judicial expert should have, before the whole analysis, forensic practitioner quantification and visual localisation of the injury

- Forensic practitioner should have technical analysis of data, for example video-records of collision between pedestrian and a vehicle, as well as physical parameters - without this documents he cannot prepare the analysis of the injuries and the whole TSCI comprehensively and qualitatively.

The idea of system FORTIS is based on necessity to use program from the first moment - first contact between patient and MD. It is reasonable to use this system for criminalistic methodology, as well as for documentation of a body injury.

\section{Conclusion}

Only skilled detective can use these methods, ways and approaches during the TCSI and that requires high knowledge of the whole issue.

Detectives should also use video-records of calculations, made in the PC Crash, as part of their accident investigation procedures. These, as "virtual elements" of evidence, enable a deeper understanding of the course of an accident beyond its verbal description. Combination of all the above mentioned facts allows a qualified assessment of the acquired knowledge and technical conclusions. The videos make it possible to "see and adopt" the casual course of an accident already as a whole, or even in the eyes of drivers, witnesses and other participants and provide the opportunity to realistically assess and judge the content of their testimonies, which creates the prerequisites for a good legal assessment of the case.

\section{References}

[1] HAVAJ P., MANDELIK J.: Criminalistic Procedures in the Process of Explaining Traffic Accidents (in Slovak). Proceedings Security in the Process of Globalization - Today and Tomorrow, Poland, 2013.

[2] MOSER, A., STEFAN, H., KASANICKY, G.: The Pedestrian Model in PC-Crash -The Introduction of a Multi Body System and its Validation. SAE TRANSACTIONS, 108, 794-802, 1999

[3] BOBROV, N., MANDELIK, J., HAVAJ, P., RABEK, V., PIWOWARSKI, J.: Possibility of the Evaluation of Injuries of an Unbelted Passenger in a Vehicle and the Need for Special Procedures while Inspecting the Site of the Accident. Security Dimension, International \& National Studies, 20, 132-164, 2016. https://doi.org/10.24356/SD/20/7

[4] MANDELIK, J.: Parameterization of pedestrian injuries and possibilities of its use in dealing with accidental (in Slovak). Dissertation thesis, University of Zilina, Institute of Judicial Engineering, 2006

[5] BOBROV, N., LONGAUER, F., MANDELIK, J.: Documentation of Fatal Road Traffic Injuries - A New Approach by FORTEST Method. Proceedings of XXth Congress of International Academy of Legal Medicine, Hungary, 2006.

[6] BOBROV, N., GINELIOVA, A., MANDELIK, J.: Trauma Injury Assessment with Survival (in Slovak). Final report of project VEGA No. 1/0428/11, 2013.

[7] BOBROV, N., GINELIOVA, A., MANDELIK, J., LONGAUER, F., MATYAS, T.: Assessing The Extent of Soft Tissue Damage in Polytrauma during Pedestrian Traffic Accidents (in Slovak). Folia Societatis Medicinae Legalis Slovacae, 2(1), 13 - 17, 2012.

[8] BOBROV, N., LONGAUER, F., SZABO, M., MANDELIK J., MANDELIKOVA, Z.: FORTIS Standardization of Traffic Injury Parameters in Forensic Medicine (in Slovak). Proceedings of scientific and professional works on the 80th anniversary of the founding of the L. Pasteur University Hospital in Kosice, Slovakia, 2004.

[9] HAVAJ, P., MANDELIK, J.: Selected Modern Methods for Solving Extraordinary Events in Road Transport (in Slovak). Monograph, Ostrowiec Swietokszyski, 2016. ISBN 978-83-64557-14-9

[10] BOBROV, N., MANDELIK, J.: Parametrization and Localization of Pedestrian Injuries by Using FORTIS System and Use of PC FORTIS. Soudni inzenyrstvi = Forensic engineering: casopis pro soudni znalectvi v technickych a ekonomickych oborech, 25(4), 227-234, 2014.

[11] BOBROV, N., MANDELIK, J., MACEJ, P.: PC Fortis program@. 